\title{
Sustained Oligoclonal T Cell Expansion Correlates with Durable Response to Immune Checkpoint Blockade in Lung Cancer
}

Sope Olugbile ${ }^{1}$, Jae-Hyun Park', Philip Hoffman ${ }^{1}$, Livia Szeto', Jyoti Patel ${ }^{1}$, Wickii T Vigneswaran ${ }^{2,3}$, Everett Vokes ${ }^{1}$, Yusuke Nakamura $^{1,2 *}$ and Kazuma Kiyotani ${ }^{1}$

${ }^{1}$ Department of Medicine, The University of Chicago, Chicago, IL, USA

${ }^{2}$ Department of Surgery, The University of Chicago, Chicago, IL, USA

${ }^{3}$ Department of Thoracic and Cardiovascular Surgery, Loyola University Medical Center, Maywood, IL, USA

\begin{abstract}
Purpose: Antibodies that target immune checkpoint molecules have demonstrated significant and durable clinical benefit through re-activation and proliferation of pre-existing tumor-infiltrating $\mathrm{CD} 8^{+} \mathrm{T}$ cells in a broad range of tumor types including lung cancer. Detailed characterization of $\mathrm{T}$ cell dynamics at clonal levels using nextgeneration T cell receptor (TCR) sequencing in large cohorts of lung cancer patients is yet reported.
\end{abstract}

Methods: We performed TCR sequencing of peripheral blood samples (and some tumors) obtained from 27 lung cancer patients undergoing single/combined immune checkpoint blockade therapies.

Results: In one responder, we found expansion of a single T cell clone (approximately $20 \%$ of the all TCR reads) in a metastatic subcutaneous lesion that showed pathologic complete response on day 17 of treatment. The same TCR CDR3 sequence was detected in $6.1 \%$ of TCR reads in the peripheral blood prior to treatment initiation and the expansion remained persistent in peripheral blood at $21.0 \%$ at week 10 and $24.3 \%$ at week 48 . In other patients who showed durable response, we also found persistent oligoclonal $\mathrm{T}$ cell expansion in their peripheral blood which was not observed in non-responders even while they remained on therapy.

Conclusion: We found sustained expansion of oligoclonal T cell clones in lung cancer patients who had durable response to immune checkpoint blockade. This suggests possible use of longitudinal TCR sequencing to assist clinical decision-making, assess synergism with other agents, and most importantly facilitate rational development of alternative treatment strategies for non-responders.

Keywords: Immune checkpoint blockade; Immunotherapy; Lung cancer; Next-generation sequencing; T cell receptor

\section{Introduction}

Following decades of skepticism, the prominent roles of an immune system in tumor control are evidently proven through the unprecedented durable response shown by immune checkpoint inhibitors in many tumor types. Characterization of the complex interactions among tumor cells, antigen-presenting cells, and effector $\mathrm{T}$ cells at molecular levels revealed key pathways in an immune signaling that mediate tumor escape from host immune surveillance. First was the discovery of a cytotoxic T lymphocyte antigen-4 (CTLA-4) molecule which was shown to down-modulate $\mathrm{T}$ cell receptor (TCR) signaling following stimulation of naïve $T$ cells $[1,2]$. Activation of anti-tumor effects through inhibition of CTLA-4 and possible mechanisms of action were later demonstrated in mouse models followed by clinical development [3-5]. Programmed death-1 (PD-1) is expressed on T cells upon their activation $[6,7]$ and plays a major role in maintenance of tolerance by interaction with its ligand PD-L1 which suppresses immune-mediated tissue damage [8]. Clinical trials of antibodies targeting the PD-1/PDL1 pathway demonstrated improvement in median overall survival when compared to conventional chemotherapies $[9,10]$ and led to approvals of nivolumab, pembrolizumab and atezolizumab for nonsmall cell lung cancer (NSCLC). However, the fact that the majority of patients did not have any clinical benefit from these agents necessitates the discovery of an accurate biomarker to guide patient selection. Both PD-L1 expression and mutational burden were suggested to be correlated with the response to immune checkpoint blockades, but the results are still controversial and their clinical use is very limited $[9,11,12]$. In the trial of first-line pembrolizumab for NSCLC, the majority of patients (55.2\%) did not respond in spite of high expression of PD-L1 (>50\%) or higher mutational burden $[12,13]$. These immune checkpoint blockades do not act directly on cancer cells, but eradicate cancer cells through the activation of cytotoxic $\mathrm{T}$ cells that target cancer cells. Hence, comprehensive longitudinal assessment of anti-tumor T cells should provide better insight into the anti-tumor effect of these agents and also may lead to the identification of factors essential for clinical benefit. Immunohistochemistry of serial biopsies obtained from responders has shown increased infiltration of $\mathrm{CD} 8^{+} \mathrm{T}$ cells in the tumor microenvironment $[14,15]$. The assessment of these processes at clonal levels of $\mathrm{T}$ cells has recently been achieved by advances in high-throughput sequencing technologies $[16,17]$, which have enabled us to identify millions of individual TCR sequences and provide the opportunity to track each of them longitudinally. Previous analyses of samples at two time-points (before and after the treatment) in a small cohort of patients revealed correlation between clonal expansion of certain T cells and clinical responses to immune checkpoint blockades [18-20]. Such findings are consistent with other reports which suggested

*Corresponding author: Yusuke Nakamura, Department of Medicine, The University of Chicago, Chicago, IL 60637, USA, Tel: 17738341405; Fax: 17737029268; E-mail: ynakamura@bsd.uchicago.edu

Received November 12, 2017; Accepted November 24, 2017; Published November 28, 2017

Citation: Olugbile S, Park J, Hoffman P, Szeto L, Patel J, et al. (2017) Sustained Oligoclonal T Cell Expansion Correlates with Durable Response to Immune Checkpoint Blockade in Lung Cancer. J Cancer Sci Ther 9: 717-722. doi:10.4172/1948-5956.1000497

Copyright: (c) 2017 Olugbile S, et al. This is an open-access article distributed under the terms of the Creative Commons Attribution License, which permits unrestricted use, distribution, and reproduction in any medium, provided the original author and source are credited. 
tumor eradication by immune checkpoint blockade through $\mathrm{T}$ cell clones targeting tumor-specific antigens [21,22]. Therefore, a detailed study of $\mathrm{T}$ cell clonal dynamics in patients with the assessment of both immediate expansion and long-term persistence in responders versus non-responders to immunotherapy could provide a valuable insight into the mechanisms that mediate tumor eradication and durable response by these agents.

\section{Materials and Methods}

\section{Patients}

We enrolled a total of 27 patients with lung cancer, who received single agent anti-PD-1 antibodies or a combined anti-PD-1 and anti-CTLA-4 antibodies, at University of Chicago Hospitals under the institutional review board (IRB) approval (\#9571). These include patients with different lung cancer subtypes (small cell and nonsmall cell) and other thoracic malignancies (thymic and large cell neuroendocrine tumors). Detailed patient information is summarized in Table 1. We analyzed 2 or more serial peripheral blood samples obtained from each patient. Blood samples $(10-20 \mathrm{~mL})$ were collected in BD Vacutainer CPT Cell Preparation tubes (BD Biosciences, San Jose, CA, USA) and peripheral blood mononuclear cells (PBMCs) were separated according to manufacturer's instructions. In patient A16.003, we also performed analyses on excess tumor samples obtained during routine clinical care as approved by IRB and with the consent

\begin{tabular}{|c|c|c|c|c|c|c|}
\hline Sample & Gender & Age & Subtype & Stage & Immunotherapy & Response \\
\hline A16.001 & Female & 45 & Squamous cell & IV & Nivolumab & NR \\
\hline A16.002 & Female & 80 & Adenocarcinoma & IV & Nivolumab & $\mathrm{R}$ \\
\hline A16.003 & Male & 80 & Squamous cell & IV & Nivolumab & $\mathrm{R}$ \\
\hline A16.007 & Female & 69 & $\begin{array}{c}\text { Adenosquamous } \\
\text { carcinoma }\end{array}$ & IV & Nivolumab & $\mathrm{R}$ \\
\hline A16.008 & Female & 70 & Adenocarcinoma & IV & Nivolumab & $\mathrm{R}$ \\
\hline A16.010 & Male & 68 & Squamous cell & IV & Nivolumab & $\mathrm{R}$ \\
\hline A16.012 & Female & 67 & Squamous cell & IV & Nivolumab & $\mathrm{R}$ \\
\hline A16.014 & Female & 40 & Adenocarcinoma & IV & Nivolumab & NR \\
\hline A16.015 & Male & 64 & Adenocarcinoma & IV & Nivolumab & NR \\
\hline A16.017 & Male & 88 & Squamous cell & IV & Nivolumab & NR \\
\hline A16.019 & Male & 62 & Adenocarcinoma & IV & Nivolumab & NR \\
\hline A16.020 & Female & 61 & Adenocarcinoma & IV & Nivolumab & $\mathrm{R}$ \\
\hline A16.021 & Male & 67 & Adenocarcinoma & IV & Nivolumab & NR \\
\hline A16.022 & Female & 43 & Adenocarcinoma & IV & Nivolumab & NR \\
\hline A16.023 & Female & 51 & Adenocarcinoma & IV & Nivolumab & NR \\
\hline A16.024 & Female & 70 & Adenocarcinoma & IV & Nivolumab & $\mathrm{R}$ \\
\hline A16.025 & Male & 59 & Adenocarcinoma & IV & Nivolumab & $\mathrm{R}$ \\
\hline A16.026 & Male & 50 & $\begin{array}{c}\text { Large cell } \\
\text { neuroendocrine } \\
\text { carcinoma from } \\
\text { thymus }\end{array}$ & IV & Nivolumab & NR \\
\hline A16.027 & Male & 60 & Adenocarcinoma & IV & Nivolumab & NR \\
\hline A16.040 & Male & 78 & Adenocarcinoma & IV & Nivolumab & NR \\
\hline A16.041 & Female & 50 & Adenocarcinoma & IV & Nivolumab & $\mathrm{R}$ \\
\hline A16.043 & Male & 75 & Adenocarcinoma & IV & Nivolumab & NR \\
\hline A16.053 & Female & 55 & Small-cell & IV & $\begin{array}{l}\text { Nivolumab/ } \\
\text { Ipilimumab }\end{array}$ & NR \\
\hline A16.055 & Male & 71 & NSCLC-NOS & IV & Nivolumab & $R$ \\
\hline A16.057 & Female & 51 & Adenocarcinoma & IV & Nivolumab & $\mathrm{R}$ \\
\hline A16.058 & Female & 62 & Squamous cell & IV & Nivolumab & NR \\
\hline .060 & Male & 53 & Small-cell & IV & $\begin{array}{l}\text { Nivolumab/ } \\
\text { Ipilimumab }\end{array}$ & NR \\
\hline
\end{tabular}

NSCLC-NOS: Non-small cell lung carcinoma-not otherwise specified; $R$ : Responder; NR: Non-Responder

Table 1: Baseline patient characteristics. of the patient. For patient A16.003 and A16.015, we separated CD8 ${ }^{+}$ and CD8 cell fraction using Dynabeads CD8 Positive Isolation Kit (Thermo Fisher Scientific, Carlsbad, CA).

\section{TCR repertoire analysis}

Total RNA was extracted from tumor/PBMC samples using RNeasy mini kit (Qiagen, Valencia, CA, USA) and TCR sequencing was performed as previously described $[17,19,23]$. Briefly, we synthesized CDNA with 5' rapid amplification of end (5'-RACE) adapter using SMART cDNA library kit (Clontech Laboratories, Mountain View, CA, USA). We then amplified the TCR cDNA with reverse primers specific for the constant regions of TCR $\alpha$ or TCR $\beta$, and a forward primer for the SMART adapter. After adding Illumina sequence adapter with barcode sequences using the Nextera XT Index kit (Illumina, San Diego, CA, USA), we performed deep sequencing by 300 -bp paired-end reads on the MiSeq platform (Illumina). We used Tcrip software for the TCR repertoire analysis as described previously [17].

\section{Results}

\section{Patient characteristics}

We analyzed TCR repertoire of 27 patients from whom we could obtain 2 or more serial samples (Table 1). All patients had stage IV diseases consisting of 16 lung adenocarcinomas, 6 squamous cell lung cancers, 2 small-cell lung cancers and 3 other thoracic malignancies. We obtained samples before immunotherapies in 13 (48.1\%) of the 27 patients and analyzed an average of 3.3 time-points per patient. In this selected patient population, $44.4 \%$ were responders (R; $12 / 27$ ) and $55.6 \%$ were non-responders (NR; 15/27), according to the RECIST criteria [24]. Among the responders, two patients (A16.003 and A16.010) did not previously receive any chemotherapy because of concerns about their capacity to tolerate therapy. The average number of previous lines of therapy was not different between the responder and non-responder groups $(\mathrm{P}=0.25 ; 1.33$ vs 1.73$)$. The distribution of lung cancer subtypes was similar between the two groups. The average age was higher among the non-responders (66.3 years) than responders (59.5 years), but not significantly different $(\mathrm{P}=0.15)$.

\section{Oligoclonal $\mathrm{T}$ cell expansion in a tumor and peripheral blood in one responder}

From a patient A16.003 with metastatic squamous cell lung cancer who had previously received palliative radiation therapy to his chest lesions (Figure 1A), we obtained biopsy of a subcutaneous lesion around the right antecubital fossa on day 17 after two cycles of anti-PD1 therapy (Figure 1B), by which time there was pathological complete response. We analyzed TCR repertoire by using our next-generation TCR sequencing method and found possible oligoclonal T-cell expansion that was reflected by a single dominant CDR3 sequence of TCR $\alpha$ and TCR $\beta$ in their sequence reads (Figure 1C). The CT scan at week 10 after receiving 4 cycles of therapy revealed significant tumor shrinkage (Figure 1D). We also analyzed TCR repertoire of peripheral blood in this patient at multiple time-points. Interestingly, even before the initiation of therapy, the peripheral blood sample of this patient possessed the TCR clonotype at frequency of $6.1 \%$ which same as was observed at the site of early pathological complete response. This specific clonotype expanded to frequency of $21.0 \%$ in week 10 and remained at the frequency of $24.3 \%$ at week 48 of the therapy (Figure 1E). We confirmed that the specific $\mathrm{T}$ cell clone was $\mathrm{CD} 8^{+} \mathrm{T}$ cells. This suggest that tumor eradication is mediated by reactivation and expansion of pre-existing tumor infiltrating $\mathrm{T}$ cell clone. 
Citation: Olugbile S, Park J, Hoffman P, Szeto L, Patel J, et al. (2017) Sustained Oligoclonal T Cell Expansion Correlates with Durable Response to Immune Checkpoint Blockade in Lung Cancer. J Cancer Sci Ther 9: 717-722. doi:10.4172/1948-5956.1000497

\section{Sustained oligoclonal $\mathrm{T}$ cell expansion in peripheral blood correlates with response}

We further analyzed TCR repertoire in peripheral blood of the patients before and after treatment initiation (Figure 2). In two responders, A16.010 (Figures 2A and 2B) and A16.057 (Figures 2C and 2D), for whom we had samples before treatment initiation, we observed oligoclonal expansion of few dominant $\mathrm{T}$ cell clones after 1 cycle of the treatment in A16.010 and after 4 cycles of the treatment in A16.057. In both A16.010 and A16.057, 1-2 dominant T cell clones detected at early time-points were sustained at week 10 of the therapy. In the other patient A16.008 (Figures 2E and 2F), although we did not have a baseline analysis, there was a single dominant $\mathrm{T}$ cell clone that remained at the high frequency over 8 months of monitoring with a

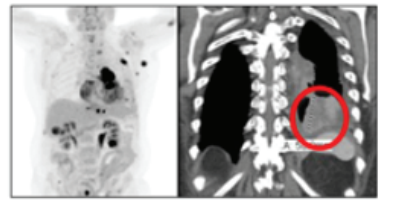

B

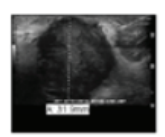

C

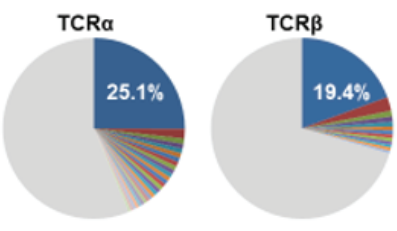

D

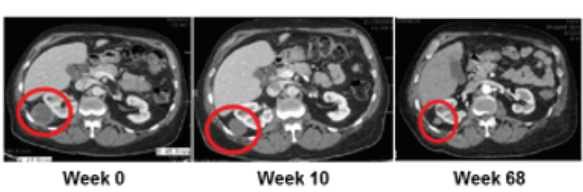

E

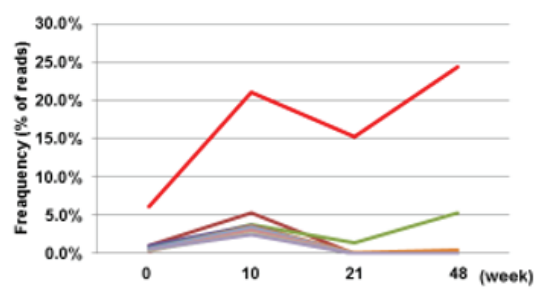

Figure 1: Presence of dominant T cell clone at metastatic site corresponds with early tumor eradication. (A-E) Patient A16.003. (A) Baseline PET scan and CT chest of patient A16.003. Both show evidence of widespread metastases in chest and abdomen. The patient received palliative radiation to chest lesions but 3 months later developed progressive disease in the abdomen (D). (B) The patient also had a $3.1 \mathrm{~cm}$ subcutaneous lesion in right antecubital fossa which was confirmed to be metastatic squamous cell cancer. (C) T cell repertoire of the right antecubital fossa lesion which was confirmed to have had complete pathological response on day 17 of therapy. Dominant clones of TCR $\alpha$ and TCR $\beta$ were observed at frequencies of $25.1 \%$ and $19.4 \%$, respectively. (D) CT scan showed response with shrinkage of right peri-renal lesion at week 10 and week 68. (E) Dynamics of top $10 \mathrm{~T}$ cell clones with highest frequencies in the peripheral blood at baseline and during the therapy. The most dominant clone found at the right antecubital fossa lesion was present in blood at $6.1 \%$ prior to initiation of immunotherapy, rose to $21.0 \%$ by week 10 and remained persistent at week 48 of therapy.

A

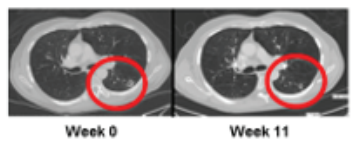

B

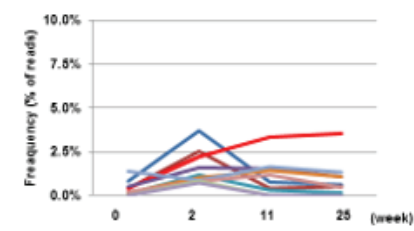

$\mathbf{E}$

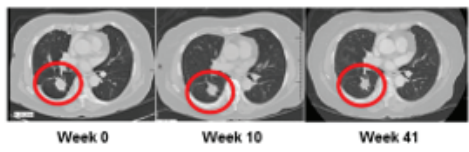

$\mathbf{F}$

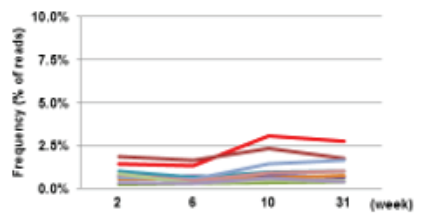

C

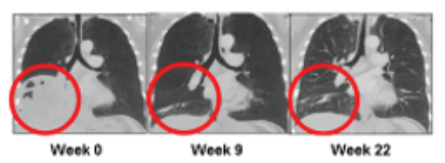

D

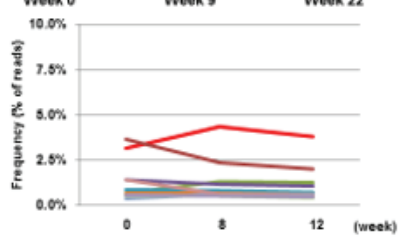

Figure 2: Early oligoclonal T cell expansion correlates with response. (A, B) Patient A16.010. His significant visceral lesion shrank on week 11 CT scan (A). TCR repertoire analysis showed that following polyclonal expansion at week 2, a T cell clone became dominant and remained persistent at high frequency at month 6 of therapy (B). (C, D) Patient A16.057. She had dramatic response to anti-PD-1 therapy as depicted by near complete resolution of right lower lobe opacity at week 9 of therapy (C). There was evidence of T cell clonal expansion in peripheral blood at week 8 after receiving 3 cycles of therapy. There was oligoclonal expansion of T cell clones (D). (E, F) Patient A16.008. The right lower lobe lesion continued to shrink at month 10 of therapy without evidence of progressive disease at other sites (E). The dominant $T$ cell clone first detected at week 2 of therapy remained dominant at high frequency at month 8 of therapy $(F)$. 
A

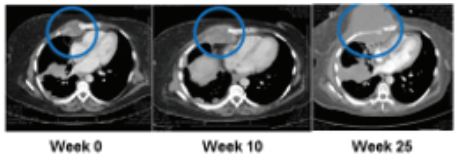

B

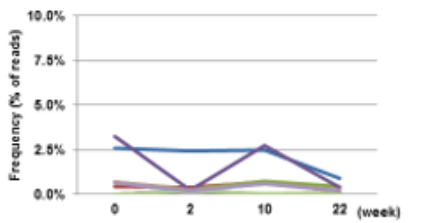

$\mathbf{E}$

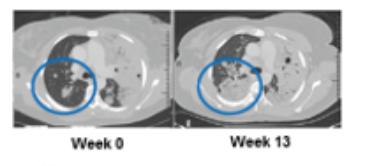

$\mathbf{F}$

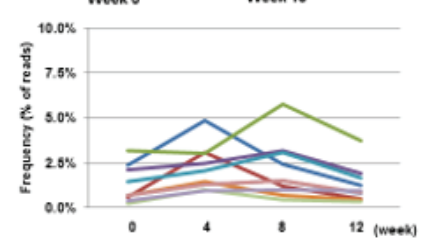

C

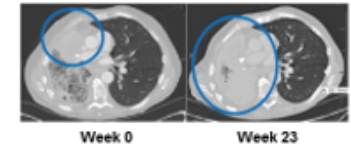

D

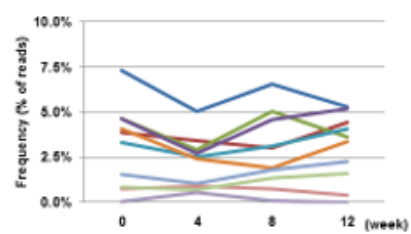

G

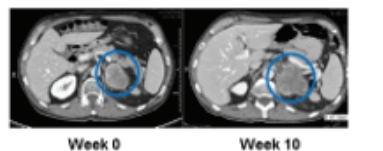

H

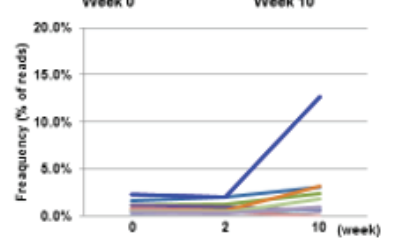

Figure 3: Absence of T cell expansion in peripheral blood correlates with resistance to immune checkpoint blockade. (A, B) Patient A16.023. Her chest wall mass continued to progress even as she remained on anti-PD1 therapy (anti-CD137 antibody was added after 4 cycles of anti-PD1 therapy as part of clinical trial) (A). TCR repertoire analysis showed no evidence of T cell clonal expansion in her peripheral blood at week 2 and week 10 (B). (C, D) Patient A16.043. He did not respond to index immunotherapy as revealed on week 23 CT scan while still on therapy, although he had previously been received anti-PD1 therapy 3 years earlier as part of clinical trial during which he had 'stable disease' (C). TCR repertoire analysis showed no evidence of clonal expansion in his peripheral blood at week 12 (D). ( $E$, F) Patient A16.022. There is evidence of progressive with increase in size of right lower lobe lesion (left lower lobe opacification persisted) (E). T cell clonal expansion was transient as most were back to baseline at week 12 of treatment $(F)$. (G, H) Patient $A 16.015$. Imaging of patient $A 16.015$ revealed progressive disease at week 10 (G), and TCR repertoire analysis showed one T cell clonal was expanded at week 10; however, this clone was CD ${ }^{8}-(H)$.

stable disease condition seen on imaging obtained at week 41. Other patients with durable response showed similar pattern of sustained dominant $\mathrm{T}$ cell clones during several months of monitoring period (Supplementary Figures $1 \mathrm{~A}-1 \mathrm{H}$ ). In contrast, non-responders did not show any dominant $\mathrm{T}$ cell clonal expansion or no persistence of dominant $\mathrm{T}$ cell clones in those we did not have baseline samples on (Figures 3A-3H) (Supplementary Figures 2A-2L). Although patient A16.015 who showed oligoclonal T cell expansion at week 10 when his tumor did not show any response to anti-PD-1 therapy, we confirmed this $\mathrm{T}$ cell clone were not $\mathrm{CD}^{+} \mathrm{T}$ cells (Figures $3 \mathrm{G}$ and $3 \mathrm{H}$ ). These data further suggest that early $\mathrm{T}$ cell clonal expansion in peripheral blood may correlate with durable clinical response.

\section{Discussion}

The elucidation of mechanisms through which tumors evade surveillance by host immune system through immune checkpoints has ushered in a new era in oncology. A subset of patients with advanced metastatic melanoma treated with agents blocking such immune checkpoints has lived beyond a decade [25]. Such significant clinical benefit has led to approvals of these agents for many tumor types. However, the fact that only a fraction of the patient population derives clinical benefits has necessitated the development of biomarkers that will guide patient selection, detect response to therapy, and determination of optimal duration of therapy. Detailed characterization of mechanisms of tumor eradication in responders at cellular levels may provide blueprint for alternative treatment strategies for non-responders. Studies in mouse models showed that these agents enhance functional activity and proliferation capability of cancer specific cytotoxic T cells [26]. The expanded T cells were demonstrated to target neoantigens generated by somatic mutations and immunization with a mutant peptide(s) was shown to cause tumor rejection similar to immune checkpoint blockade [21]. These findings were further supported by a report showing that a responder to a CTLA-4 blockade had T cell clonal expansion targeting a neoantigen [22]. Thus, it is likely that tumor eradication by these agents is through reactivation and proliferation of pre-existing tumor infiltrating cancerspecific cytotoxic $\mathrm{T}$ cells. This is concordant with the results that $e x-$ vivo expansion and adoptive transfer of mutant-specific $\mathrm{T}$ cell clones achieved durable clinical response in different tumor types [27,28]. Hence, longitudinal comprehensive analyses of $\mathrm{T}$ cell dynamics both at the tumor site(s) and in peripheral blood may elucidate the footprints of tumor eradicating $\mathrm{T}$ cell dynamics in responders. Recent advances in the next-generation sequencing have provided us tools to measure dynamic changes of immune cells including individual $\mathrm{T}$ cell clones within tumors and peripheral blood samples [27]. In our patient cohort, we found a patient who had a single dominant $\mathrm{T}$ cell clone with the TCRa $\beta$ frequencies of around $20 \%$ (Figure 1C) at a metastatic site with pathological complete response at day 17 of the therapy. The same $\mathrm{T}$ cell clone was found in peripheral blood at the frequency of $6.1 \%$ before the treatment and was expanded to $21.0 \%$ at week 10 . This frequency is similar to that reported in a melanoma patient who showed complete radiological response [20], thus monitoring $\mathrm{T}$ cell clonal dynamics using this comprehensive approach (i.e. analyses of entire repertoire) may reflect response to therapy more accurately. Our data also suggest that oligoclonal $\mathrm{T}$ cell clonal expansion after one or a few cycles of therapy may be an early sign of favorable clinical response. Although we need a larger number of samples at multiple fixed timepoints in comparison to the baseline pretreatment sample and further confirmation that those expanded $\mathrm{T}$ cells are reactive to cancer cells, monitoring $\mathrm{T}$ cell repertoire in peripheral blood could expedite clinical decision making in neoadjuvant and oligometastatic disease settings. Patients with evidence of early oligoclonal $\mathrm{T}$ cell expansion may remain on therapy while those without clonal $\mathrm{T}$ cell expansion may proceed to surgery or start on alternative therapies. Similar to previous 
reports in mouse models and in human adoptive $\mathrm{T}$ cell transfer therapies $[21,27,28]$, we found that $1-2$ dominant $\mathrm{T}$ cell clones may be responsible for tumor eradication in responders and their persistence at high frequencies correlate with durable response (Figures 1and 2). Even in the patients for whom we did not have baseline pre-treatment samples but who had durable response, we observed the persistence of 1-2 dominant $\mathrm{T}$ cell clones in their peripheral blood for many months (Supplementary Figure 1). This further supports previous observations that only a subset of patients that had adoptive $\mathrm{T}$ cell therapy had durable response and in them (unlike non-responders) the re-infused $\mathrm{T}$ cell clones following ex-vivo expansion remained at high frequencies for several months afterwards [27,28]. So also a recent report demonstrated that non-responders to immunotherapy actually had $\mathrm{T}$ cell clones targeting neoantigens, but yet did not derive clinical benefit like as a result of the inability of these $\mathrm{T}$ cell clones to proliferate [29]. These together suggest that in addition to having cancer-specific tumor infiltrating $\mathrm{T}$ cells, it is also critically important for these $\mathrm{T}$ cells to have self-renewal and sustained expansion capacities. The response rate in this study cohort did not reflect correctly in an entire clinic since we included patients whom we already knew to have durable response.

\section{Conclusion and Limitations}

The limitations of our study include our inability to identify antigens targeted by the expanded $\mathrm{T}$ cell clones due to unavailability of tissue samples for tumor exome sequencing. We cannot exclude a possibility that some of the $\mathrm{T}$ cell clonal expansions may be caused by other factors (e.g. infections). However, the pattern of $\mathrm{T}$ cell clonal expansion by infection would be typically rapid followed by contraction once the infection is controlled. In addition, we were unable to apply any statistical analyses to evaluate significant differences between responders and non-responders because i) we only had three responders of whom we had baseline pre-treatment samples, ii) the time-points of sample collection varied among patients for logistical reasons, and then iii) we did not observe any correlation between tumor burden and clonal frequencies. We also need to consider immune suppression mechanisms in cancer cells using a large cohort set of patients. To the best of our knowledge, this is the largest report of longitudinal TCR sequencing of peripheral blood in patients treated with immunotherapy. It is also the first to include multiple time-points during the course of treatment from the first treatment to as far as 13 months (although we had no baseline samples in some of these cases). It is notable that early and sustained oligoclonal $\mathrm{T}$ cell expansion may be correlate with better durable response to immune checkpoint blockades and may provide deeper insights into mechanisms of tumor eradication by these agents.

\section{Conflict of Interest}

Y.N. is a stockholder and an adviser of OncoTherapy Science Inc. J.P. is a scientific adviser of OncoTherapy Science Inc. Other authors have no conflict of interest concerning this study.

\section{Acknowledgments}

The authors would like to thank Makiko Harada for excellent technical assistance. We would like to thank Drs. Rui Yamaguchi, Seiya Imoto and Satoru Miyano at the University of Tokyo for developing the algorithm of TCR repertoire analysis and helpful supports in data management. The super-computing resource (http://sc.hgc.jp/shirokane.html) was provided by Human Genome Center, the Institute of Medical Science, the University of Tokyo.

\section{References}

1. Brunet JF, Denizot F, Luciani MF, Roux-Dosseto M, Suzan M, et al. (1987) A new member of the immunoglobulin superfamily--CTLA-4. Nature 328: 267 270

2. Alegre ML, Noel PJ, Eisfelder BJ, Chuang E, Clark MR, et al. (1996) Regulation of surface and intracellular expression of CTLA4 on mouse T cells. J Immunol 157: 4762-4770.

3. Mokyr MB, Kalinichenko T, Gorelik L, Bluestone JA (1998) Realization of the therapeutic potential of CTLA-4 blockade in low-dose chemotherapy-treated tumor-bearing mice. Cancer Res 58: 5301-5304

4. Hurwitz AA, Yu TF, Leach DR, Allison JP (1998) CTLA-4 blockade synergizes with tumor-derived granulocyte-macrophage colony-stimulating factor for treatment of an experimental mammary carcinoma. Proc Natl Acad Sci USA 95: 10067-10071.

5. Hodi FS, Mihm MC, Soiffer RJ, Haluska FG, Butler M, et al. (2003) Biologic activity of cytotoxic $T$ lymphocyte-associated antigen 4 antibody blockade in previously vaccinated metastatic melanoma and ovarian carcinoma patients. Proc Natl Acad Sci USA 100: 4712-4717.

6. Ishida Y, Agata Y, Shibahara K, Honjo T (1992) Induced expression of PD-1, novel member of the immunoglobulin gene superfamily, upon programmed cell death. EMBO J 11: 3887-3895.

7. Nishimura H, Agata Y, Kawasaki A, Sato M, Imamura S, et al. (1996) Developmentally regulated expression of the PD-1 protein on the surface of double-negative (CD4-CD8-) thymocytes. Int Immunol 8: 773-780.

8. Freeman GJ, Long AJ, Iwai Y, Bourque K, Chernova T, et al. (2000) Engagement of the PD-1 immunoinhibitory receptor by a novel B7 family member leads to negative regulation of lymphocyte activation. J Exp Med 192: 1027-1034.

9. Brahmer JR, Drake CG, Wollner I, Powderly JD, Picus J, et al. (2010) Phase I study of single-agent anti-programmed death-1 (MDX-1106) in refractory solid tumors: Safety, clinical activity, pharmacodynamics, and immunologic correlates. J Clin Oncol 28: 3167-3175.

10. Hamid O, Robert C, Daud A, Hodi FS, Hwu WJ, et al. (2013) Safety and tumo responses with lambrolizumab (anti-PD-1) in melanoma. N Engl J Med 369: 134-144.

11. Topalian SL, Hodi FS, Brahmer JR, Gettinger SN, Smith DC, et al. (2012) Safety, activity, and immune correlates of anti-PD-1 antibody in cancer. N Engl J Med 366: 2443-2454.

12. Rizvi NA, Hellmann MD, Snyder A, Kvistborg P, Makarov V, et al. (2015) Mutational landscape determines sensitivity to PD-1 blockade in non-small cel lung cancer. Science 348: 124-128.

13. Reck M, Rodriguez-Abreu D, Robinson AG, Hui R, Csoszi T, et al. (2016) Pembrolizumab versus chemotherapy for PD-L1-positive non-small-cell lung cancer. N Engl J Med 375: 1823-1833.

14. Nghiem PT, Bhatia S, Lipson EJ, Kudchadkar RR, Miller NJ, et al. (2016) PD-1 blockade with pembrolizumab in advanced merkel-cell carcinoma. N Engl J Med 374: 2542-2552

15. Herbst RS, Soria JC, Kowanetz M, Fine GD, Hamid O, et al. (2014) Predictive correlates of response to the anti-PD-L1 antibody MPDL3280A in cancer patients. Nature 515: 563-567.

16. Freeman JD, Warren RL, Webb JR, Nelson BH, Holt RA (2009) Profiling the $\mathrm{T}$-cell receptor beta-chain repertoire by massively parallel sequencing. Genome Res 19: 1817-1824

17. Fang H, Yamaguchi R, Liu X, Daigo Y, Yew PY, et al. (2015) Quantitative T cell repertoire analysis by deep cDNA sequencing of $\mathrm{T}$ cell receptor alpha and beta chains using next-generation sequencing (NGS). Oncoimmunology 3 : e968467.

18. Tumeh PC, Harview CL, Yearley JH, Shintaku IP, Taylor EJ, et al. (2014) PD-1 blockade induces responses by inhibiting adaptive immune resistance. Nature 515: 568-571.

19. Inoue H, Park JH, Kiyotani K, Zewde M, Miyashita A, et al. (2016) Intratumora expression levels of PD-L1, GZMA, and HLA-A along with oligoclonal T cell expansion associate with response to nivolumab in metastatic melanoma. Oncoimmunology 5: e1204507.

20. Haymaker CL, Kim D, Uemura M, Vence LM, Phillip A, et al. (2017) Metastatic melanoma patient had a complete response with clonal expansion after whole brain radiation and PD-1 blockade. Cancer Immunol Res 5: 100-105.

21. Gubin MM, Zhang X, Schuster H, Caron E, Ward JP, et al. (2014) Checkpoint 
Citation: Olugbile S, Park J, Hoffman P, Szeto L, Patel J, et al. (2017) Sustained Oligoclonal T Cell Expansion Correlates with Durable Response to Immune Checkpoint Blockade in Lung Cancer. J Cancer Sci Ther 9: 717-722. doi:10.4172/1948-5956.1000497

blockade cancer immunotherapy targets tumour-specific mutant antigens. Nature 515: 577-581.

22. Van Rooij N, Van Buuren MM, Philips D, Velds A, Toebes M, et al. (2013) Tumor exome analysis reveals neoantigen-specific T-cell reactivity in an ipilimumabresponsive melanoma. J Clin Oncol 31: e439-442.

23. Choudhury NJ, Kiyotani K, Yap KL, Campanile A, Antic T, et al. (2015) Low T-cell receptor diversity, high somatic mutation burden, and high neoantigen load as predictors of clinical outcome in muscle-invasive bladder cancer. Eur Urol Focus 2: 445-452.

24. Eisenhauer EA, Therasse P, Bogaerts J, Schwartz LH, Sargent D, et al. (2009) New response evaluation criteria in solid tumours: revised RECIST guideline (version 1.1). Eur J Cancer 45: 228-247.

25. Schadendorf D, Hodi FS, Robert C, Weber JS, Margolin K, et al. (2015) Pooled analysis of long-term survival data from phase II and phase III trials of ipilimumab in unresectable or metastatic melanoma. J Clin Oncol 33: 1889-1894.

26. Wong RM, Scotland RR, Lau RL, Wang C, Korman AJ, et al. (2007) Programmed death-1 blockade enhances expansion and functional capacity of human melanoma antigen-specific CTLs. Int Immunol 19: 1223-1234.

27. Tran E, Robbins PF, Lu YC, Prickett TD, Gartner JJ, et al. (2016) T-cell transfer therapy targeting mutant KRAS in cancer. N Engl J Med 375: 2255-2262.

28. Tran E, Turcotte S, Gros A, Robbins PF, Lu YC, et al. (2014) Cancer immunotherapy based on mutation-specific CD4+ T cells in a patient with epithelial cancer. Science 344: 641-645.

29. Anagnostou V, Smith KN, Forde PM, Niknafs N, Bhattacharya R, et al. (2016) Evolution of neoantigen landscape during immune checkpoint blockade in nonsmall cell lung cancer. Cancer Discov 7: 264-276. 